\title{
Algunas falacias respecto a las políticas de autoconstrucción en los países en desarrollo
}

\author{
Rod Burgess*
}

En los últimos años los principales organismos financieros internacionales han dado preferencia a los proyectos de lotes y servicios de mejoramiento de tugurios y de asentamientos irregulares; este artículo tiene como objetivo confrontar la experiencia obtenida en dichos proyectos y las discusiones teóricas sobre la autoconstrucción de vivienda que surgen de ese enfoque. Se trata de demostrar que dichas políticas dan lugar a mitos y falacias que tienden a ocultar la verdadera naturaleza de los problemas que surgen en los proyectos. Se mencionan como ejemplos de dichas falacias: que el interés de los organismos internacionales en las estrategias de autoconstrucción no es nuevo; que no es posible afirmar que la política de autoconstrucción ha contribuido en forma determinante a solucionar el problema de la vivienda en los países menos desarrollados; asimismo, tampoco es factible asegurar sin iugor a dudas que ios proyectos de autoconstrucción han beneficiado en gran medida a los grupos más pobres; y, tampoco puede establecer que los proyectos de lotes y servicios y de mejoramiento se caracterizan por la utilización de mano de obra familiar no remunerada.

Hace ya más de diez años que los principales organismos internacionales relacionados con el financiamiento de la vivienda y el desarrollo urbano en los países del Tercer Mundo cambiaron su política de promover proyectos "convencionales" para grupos de medianos y altos ingresos por la de proveer vivienda económica y programas de mejorarmiento basados en los principios de autoconstrucción y desarrollo gradual para grupos de "bajos ingresos". Tal cambio ha provocado también la reorientación de algunas políticas nacionales de vivienda hacia ese tipo de enfoque. Muchos de los proyectos de lotes y servicios y de mejoramiento de tugurios y de asentamientos irregulares surgidos de las nuevas políticas fueron terminados hace tiempo, otros están cerca de su terminación, y muchos más se encuentran en la etapa de planeación. Llegó el momento, por consiguiente, de establecer la relación entre los datos acerca de la experiencia derivada de esos proyectos y las discusiones teóricas sobre la autoconstrucción de vivienda que acompañaron a esos cambios de políticas.

* Departamento de Geografía e Ingeniería del Medio Ambiente (The Department of Geography and Environmental Engineering), Universidad Johns Hopkins, Baltimore, Maryland, 21218. Traducción del inglés: Mario Zamudio. 
Como veremos, este trabajo revela varios mitos y falacias con respecto a esas polfticas; mitos y falacias que tienden a velar la verdadera naturaleza de los problemas que surgen en los proyectos a los que dichas políticas dan origen.

En el marco de este trabajo no es posible un análisis exhaustivo del tema, pero remitimos al lector al libro Labour, Shelter and Global Capitalism (Mano de obra, vivienda y capitalismo global), ${ }^{1}$ el cual aborda esos datos empíricos con mucho mayor detalle, en el contexto de un análisis crítica de la versión que han adoptado los sistemas mundiales sobre la teoría de la reproducción de la fuerza de trabajo, en cuanto explicación teórica del fenómeno del apoyo internacional a la autoconstrucción de vivienda.

Primera falacia: que la actual ola de interés de los organismos internacionales y gobiernos nacionales en las estrategias de autoconstrucción de viviendas constituye una tendencia nueva

Si bien la participación actual de los gobiernos nacionales y los organismos internacionales excede con mucho la de periodos anteriores, el apoyo a la autoconstrucción de viviendas data de finales de los años cuarenta. El primer ejemplo de un interés sostenido en proyectos de autoconstrucción de viviendas para América Latina lo constituye la participación del gobierno estadunidense en el "financiamiento de la autoconstrucción de vivienda" para trabajadores rurales en Puerto Rico. La construcción de viviendas se inició en 1949, y hacia principios de los años sesenta se habían completado entre 30000 y 40000 casas -en la mayoría de los casos se trataba de "pies de casa" que habrían de ampliarse mediante un desarrollo gradual.

En los años sesenta, el Banco Mundial participó en el suministro de fondos para un número limitado de proyectos de abastecimiento urbano de agua y drenaje, que dio como resultado el mejoramiento de algunos asentamientos populares. ${ }^{2}$ Después de 1965, el Fondo de las Naciones Unidas para el Desarrollo también ofreció financiamiento y ayuda técnica para la autoconstrucción de viviendas experimentales de bajo costo, asi como para la rehabilitación de asentamientos irregulares, si bien las sumas comprometidas fueron relativamente reducidas. Además, durante ese periodo algunos gobiernos contaban con programas de autoconstrucción de viviendas como parte de sus políticas nacionales de vivienda (por ejemplo,

${ }^{1}$ R. Burgess, Labour, Shelter and Global Capitalism, Metheum, Londres, 1987.

2 S. Blitzer, J. E. Hardoy y D. Satterthwaite, "The Sectoral and Spatial Distribution of Multilateral Aid for Human Settlements", en Habitat International, vol. 7, núm. 1/2, 1983, pp. 103-127. 
Colombia, Kenia y Zambia). Payne ${ }^{3}$ también consigna pruebas de que ya en los años cincuenta en algunos países (Perú, Indonesia y Turquía) se hicieron esfuerzos, aunque aislados y reducidos, para la mejora de asentamientos.

Con todo, los más importantes fueron los sustanciales compromisos de ayuda bilateral (USAID) y multilateral (Banco Interamericano de Desarrollo: BID) para proyectos de "financiamiento de la autoconstrucción" de vivienda y préstamos para el mejoramiento de casas en varios países africanos y latinoamericanos, a principios de los años sesenta. A través de convenios establecidos como parte de la Alianza para el Progreso, USAID aportó una fuerte ayuda financiera destinada a los grupos de bajos ingresos en la forma de préstamos para el mejoramiento habitacional y la edificación de viviendas basada en la autoconstrucción con la ayuda o cooperación familiar.

De manera similar, el interés del BID en el financiamiento de viviendas mediante la autoconstrucción, propiamente dicha, y la autoconstrucción con ayuda mutua, data de un convenio firmado entre el gobierno estadunidense y el Banco en 1961, en el que se estableció el Fideicomiso para el Progreso Social (FPS) (Social Progress Trust Fund: SPTF) como parte de los convenios de la Alianza para el Progreso. Así, el Fideicomiso pudo disponer de financiamientos por un valor de 525 millones de dólares para la concesión de préstamos privilegiados en los "campos de asentamiento y mejoras en los usos del suelo, vivienda para grupos de bajos ingresos, abastecimiento de agua e higiene pública, así como de instalaciones para la educación superior". ${ }^{4}$

Junto con esos fondos, el BID aprobó 29 proyectos de vivienda urbana, 33 proyectos de agua y drenaje y muchos programas para la construcción de mercados, escuelas e instalaciones de salud pública en áreas urbanas. Muchos de esos proyectos de vivienda y urbanización incluían la aplicación de sistemas de construcción mediante el "financiamiento de la autoconstrucción" y la "ayuda mutua", así como un número importante de préstamos para el mejoramiento habitacional. En esa época, Chile, Colombia y Perú participaron en proyectos de ese tipo financiado por el BID, y a menu do el resultado fue satisfactorio.

Los préstamos del BID, de USAID y del FPS de la Alianza para el Progreso entregados al organismo nacional de vivienda de Colombia (ICT) le permitieron construir unas 50000 casas entre 1961 y 1963. La mayor parte de ellas se construyó mediante el sistema de financiamiento de la autoconstrucción; tan es así, que para $1967,45 \%$ de todas las casas producidas por el ICT desde su fundación, a principios de los años cuarenta, había sido construido aplicando ese sistema.

${ }^{3}$ G. K. Payne (ed.), Low Income HousIng In the Developing World - The Hole of Sites and Ser vices and Settlement Upgrading, John Wiley and Sons, Chichester, 1984.

${ }^{4}$ Véase la nota 2. 
Lo interesante es que, a pesar de las actividades de proselitismo de un creciente número de expertos, el interés de los organismos internacionales y nacionales en esos programas decayó en la segunda mitad de los años sesenta. Por ejemplo, una vez que los recursos del FPS quedaron totalmente comprometidos en 1966, el interés del BID en los proyectos de autoconstrucción y mejoramiento decayó, y USAID desvió su atención hacia el fortalecimiento o la creación de instituciones para la vivienda, la urbanización y financieras; en particular de asociaciones de ahorro y préstamo cuyos esfuerzos eran destinados en gran medida a las familias de ingresos medios y superiores.

La actual y más sostenida ola de interés de los organismos internacionales y los gobiernos nacionales en las actividades de autoconstrucción de viviendas se inició en 1973, cuando el Banco Mundial intentó reorientar sus políticas habitacibnales hacia los grupos de bajos ingresos, mediante el patrocinio de proyectos de lotes y servicios y mejoramiento de barriadas basados en los tres principios de desarrollo gradual, contribución de mano de obra propia para la construcción y reducción de las normas mínimas. Para mediados de los años setenta, la mayoría de los demás organismos internacionales (tanto bilaterales como multilaterales) que participaban en el financiamiento de proyectos relacionados con la vivienda urbana habían cambiado sus políticas de vivienda en un sentido similar.

De esta breve reseña surge un buen número de consideraciones teóricas, que no pueden ser analizadas en este trabajo, pero que ya han sido discutidas exhaustivamente en otros; entre ellas, la relación entre la asesoría pericial y los intereses materiales en la determinación de las políticas de vivienda y entre los organismos de ayuda bilateral y multilateral y los intereses de los gobiernos y países donadores y receptores. ${ }^{5}$ Es evidente, no obstante, que entre los dos periodos existen diferencias significativas en la naturaleza de las políticas de autoconstrucción de viviendas: 1) durante el primer periodo predominó el apoyo de los organismos bilaterales, mientras que en el periodo actual ha predominado el de los organismos multilaterales; 2) en el primer periodo, los esfuerzos se concentraron en la construcción de viviendas nuevas, mientras que, en el actual, la mayor parte de los recursos ha sido dirigida hacia el mejoramiento de viviendas existentes; 3) en el primer periodo se puso un mayor énfasis en la ayuda "mutua", antes bien que en los sistemas de construcción de los años recientes, basados en la familia, y 4) quizá lo más importante de todo, la primera fase de las políticas de autoconstrucción de vivienda ocurrió durante un periodo de expansión económica mundial y no en uno de recesión.

Podría argumentarse que la falta de claridad y preocupación en la historia incial de las políticas de autoconstrucción de viviendas ha llevado, en

5 Véase la nota 1. 
parte, a una subestimación de la importancia de los elementos políticos en la determinación del volumen y distribución de la ayuda que para esos propósitos han dado los organismos internacionales a cada uno de los países. Es muy probable que la primera ola de ayuda internacional para la autoconstrucción de viviendas en América Latina, a principios de los años sesenta, haya sido motivada meramente por consideraciones políticas.

Muchos de los recursos para esos proyectos se derivaron de los convenios de la Alianza para el Progreso, y en la actualidad se reconoce ampliamente que la Alianza para el Progreso fue preparada, de manera esencial, para asegurar la estabilidad política del continente como resultado de la Revolución Cubana, y que decayó una vez que el tema de la seguridad perdió su urgencia:

Incluso la Alianza para el Progreso, establecida a principios de los años sesenta con tantos bombos y platillos y tan noble retórica respecto al fomento del desarrollo económico latinoamericano, fue formulada en realidad como una respuesta directa al ascenso de Fidel Castro en Cuba y a la creencia en la amenaza de tomas de poder comunistas en otros países latinoamericanos. ${ }^{6}$

La existencia en esa época de programas para la autoconstrucción de viviendas en gran escala en América Latina debe, sin duda, ser motivo de reflexión para quienes argumentan que la cuestión del apoyo internacional a la autoconstrucción de viviendas puede ser comprendida únicamente en función de procesos económicos (como la recesión en la economía capitalista mundial). Sea cual fuere el caso, es evidente que una mayor investigación de la historia de las políticas de autoconstrucción de viviendas ayudaría enormemente en los debates teóricos en torno a las razones del actual au mento del interés en esas políticas y del apoyo dado a las mismas.

Segunda falacia: que las políticas de autoconstrucción de viviendas han contribuido de manera significativa a la solución de los problemas de vivienda de los países menos desarrollados

En una gran mayoría de las obras publicadas sobre las políticas de autoconstrucción de viviendas de los países en desarrollo, se encuentra implícita la noción de que los gobiernos nacionales y los organismos internacionales han hecho un importante intento por atender mediante esas políticas los graves problemas habitacionales que enfrentan esos países. Pero, ¿qué pruebas existen de esa opinión? Evidentemente, tal punto de vista debería 
estar basado en una evaluación de la participación financiera de los organismos internacionales en dichas políticas, tanto en términos absolutos como relativos. Esto es, en función de la importancia sectorial de su participación financiera en la vivienda urbana en relación con todas las participaciones financieras sectoriales; en la relación entre, por una parte, el volumen y el tipo de viviendas resultantes y, por la otra, la necesidad de viviendas; $\mathrm{y}$, finalmente, en la importancia relativa de las partidas gubernamentales nacionales destinadas a la autoconstrucción de viviendas respecto a las partidas para viviendas de todos tipos.

Para llevar a cabo tal análisis, existe un gran número de dificultades prácticas que se relacionan con los problemas que plantean el concepto y la definición de las actividades de autoconstrucción de viviendas; la dimensión de la necesidad de viviendas; y la presentación de datos y categorías en los que se agregan tanto las actividades de autoconstrucción como las que no lo son. A pesar de todas estas dificultades, los datos indican que el apoyo financiero de los organismos internacionales a las actividades de autoconstrucción ha permanecido relativamente bajo desde el punto de vista sectorial; que las políticas de autoconstrucción no han reducido mucho la creciente necesidad de viviendas, sino que, en realidad los problemas han empeorado; y que, a nivel nacional, los programas de autoconstrucción han seguido constituyendo un componente relativamente pequeño del gasto nacional total destinado a las actividades de construcción de vivienda urbana.

Es muy difícil determinar con precisión la extensión de los préstamos internacionales para proyectos de lotes y servicios y mejoramiento de barriadas. Debido a la manera como son presentados los datos, todas las estimaciones del valor de los préstamos o del volumen de la producción tienen que ser, necesariamente, aproximativas.

Desde los años setenta, el principal apoyo para ésas y otras actividades relacionadas con la vivienda urbana ha venido de fuentes multilaterales, y el Banco Mundial es, con mucho, el mayor prestador de fondos para las actividades de autoconstrucción de viviendas. Los préstamos para vivienda urbana (que a partir de 1972 han consistido principalmente en proyectos de lotes y servicios y de mejoramiento) se encuentran incorporados en el sector de "desarrollo urbano", que también incluye préstamos para transporte y proyectos de urbanización. Además, el Banco cuenta también con dos líneas de crédito sectorial que, ya sea directa o indirectamente, se relacionan con actividades de autoconstrucción de viviendas, de abastecimiento de agua y drenaje y para empresas de mediana escala (después de 1980).

Aunque los préstamos para estos dos últimos sectores se destinan abrumadoramente a las zonas urbanas, también existen algunos desponibles para las zonas rurales. No obstante, si agregamos los datos de esos tres sectores, podemos obtener una idea apoximada de la extensión de la participación financiera del Banco Mundial en los proyectos de vivienda urbana que han 
contado con un componente de autoconstrucción. El cuadro 1 consigna los datos para el periodo 1973-1983, esto es, desde que el Banco desvió sus políticas urbanas hacia la autoconstrucción de viviendas y desarrollo gradual. Durante ese periodo, el Banco prestó un total de 97004.8 millones de dólares para todos los sectores. De ellos, 9872.2 millones (9.6\%) fueron prestados para los tres sectores relacionados con la vivienda urbana; $2936.8 \mathrm{mi}$ llones (3.0\% del total para todos los sectores) fueron destinados al desarrollo urbano; 5044.6 millones (5.2\%) a proyectos de abastecimiento de agua y drenaje; y 1305.8 millones (1.4\%), a empresas de mediano tamaño.

CUADRO 1

Operaciones de préstamos del Banco Mundial para sectores relacionados con la vivienda urbana (1973-1983)

\begin{tabular}{lcc}
\hline Sector & $\begin{array}{c}\text { Total en millones } \\
\text { de dls. de E. U. }\end{array}$ & $\begin{array}{c}\text { Porcentaje } \\
\text { del total }\end{array}$ \\
\hline $\begin{array}{l}\text { Desarrollo urbano } \\
\text { Agua y drenaje } \\
\begin{array}{c}\text { Empresas de } \\
\text { mediano tamaño }\end{array}\end{array}$ & 2936.8 & 3.0 \\
$\begin{array}{c}\text { Total relacionado } \\
\text { con la vivienda } \\
\text { urbana }\end{array}$ & 5044.6 & 5.2 \\
$\begin{array}{l}\text { Total de todos los } \\
\text { sectores }\end{array}$ & 1305.8 & 1.3 \\
\hline
\end{tabular}

Fuente: World Bank Annual Reports, 1973-1983. ${ }^{7}$

Cuando se recuerda que la categoría "desarrollo urbano" incluye también préstamos para otros tipos de proyectos de vivienda, transporte urbano y urbanización, puede verse que la asignación sectorial para proyectos de lotes y servicios y de mejoramiento ha sido limitada. Cohen, ${ }^{8}$ funcionario del Banco Mundial, estima que entre 1972 y 1982 el Banco prestó aproximadamente 2000 millones de dólares a 36 gobiernos para financiar 62 proyectos urbanos, 36 de los cuales fueron proyectos de vivienda urbana.

Los otros organismos multilaterales probablemente han otorgado aún menor prioridad a esos tipos de proyectos de autoconstrucción, mientras que han tendido a favorecer grandes proyectos de abastecimiento de agua y drenaje o soluciones más convencionales de vivienda para grupos de medianos ingresos. Blitzer, Hardoy y Satterthwaite ${ }^{9}$ han identificado 15 orga-

${ }^{7}$ World Bank Anuual Reports, Washington, D. C., 1973-1983.

${ }^{8}$ M. A. Cohen, The Challenge of Repiicability: Towards a New Paradigm for Urban Shelter in Developing Countries, World Bank Reprint Series núm. 287, World Bank, Washington, D. C., 1983.

${ }^{9}$ Véase la nota 2. 
nismos multilaterales que operan en el campo de la vivienda urbana, pero apenas cuatro de ellos (Banco Mundial, Banco Africano de Desarrollo, Banco Interamericano de Desarrollo y Banco Asiático de Desarrollo) representaron $80.4 \%$ de todos los préstamos para desarrollo urbano y $91.4 \%$ de préstamos para abastecimiento de agua y drenaje, otorgados por los 15 organismos durante el periodo 1970-1981.

Esos cuatro organismos prestaron 7700 millones de dólares para desarrollo urbano y abastecimiento de agua y drenaje durante el periodo. Sólo 2400 millones de dólares se destinaron al desarrollo urbano, y esta categoría incluyó también proyectos de urbanización y de transporte urbano y algunos proyectos de vivienda convencional (particularmente durante el periodo 1970-1973). Tomando en cuenta estas proporciones, se puede calcular que durante el periodo 1970-1981 los préstamos multilaterales para proyectos de desarrollo urbano se hicieron a un promedio de 247.4 millones de dólares por año y los préstamos para agua y drenaje a un promedio de 488.1 millones por año, lo que da una participación financiera anual promedio total de 735.5 millones de dólares.

La mayoría de los préstamos multilaterales incluían la obligación del gobierno receptor de contribuir a los costos de los proyectos, y la contribución fue generalmente de alrededor de $45 \%$ del costo total de los proyectos. Sobre esta base, se puede estimar que, durante los años setenta, el gasto global total en proyectos de base multilateral fue de 449.8 millones de dólares por año para desarrollo urbano y de 887.5 millones por año para abastecimiento de agua y drenaje. Lo anterior arroja un total de 1337.3 millones de dólares por año para esas dos categorías de proyectos relacionados con la vivienda urbana.

Es aún más difícil obtener estimaciones precisas de la extensión de las participaciones financieras bilaterales en este campo. Los datos de la OECD (DAC) revelan que, durante el periodo 1980-1983, alrededor de 17\% del total de la participación bilateral se destinó al desarrollo de servicios públicos (con la exclusión del desarrollo de la energía) y alrededor de $4 \%$ a la infraestructura y el bienestar sociales -aproximadamente 668 y 284 millones de dólares por año, respectivamente-. Es claro que estas dos categorías incluyen muchos proyectos que no se relacionan directamente con los de vivienda urbana (por ejemplo, proyectos de urbanización y de transporte urbano, terminales de transportes, oleoductos, infraestructura industrial, etc.], y que la proporción de esos préstamos, que se destinó a programas de lotes y servicios, mejoramiento y abastecimiento de agua y drenaje, debe de haber sido relativamente baja.

En un reciente seminario sobre la ayuda bilateral europea para asentamientos humanos, se llegó a la conclusión de que sólo una reducida proporción de la misma tiene como objetivo esos tipos de proyectos. Se calculó que menos de $5 \%$ de la ayuda inglesa y francesa se destinaba a proyectos de vivienda, agua y drenaje para grupos de bajos ingresos, y que los orga- 
nismos bilaterales holandeses, suecos y suizos destinaban menos de $15 \%$ de su ayuda a esos propósitos.

El único gobierno con un programa de ayuda importante destinado específicamente al suministro de viviendas para grupos de bajos ingresos es USAiD, pero incluso en este caso se estima que el organismo no gastó más de 100 millones de dólares anuales a finales de los años setenta en proyectos de lotes y servicios, de "pies de casa" y de mejoramiento. ${ }^{10}$ La proporción de préstamos bilaterales provenientes de la OPEP y destinados a proyectos de lotes y servicios y de mejoramiento ha sido insignificante, y es sumamente dudoso que los préstamos de esta fuente para agua y drenaje superen los 250 millones de dólares por año.

Así, si se excluye la contribución del gobierno receptor, se puede hacer una estimación muy aproximada del total de la participación multilateral y bilateral en las actividades relacionadas con la vivienda urbana en los últimos años del decenio de los setenta y principios de los ochenta: los préstamos para desarrollo urbano fueron aproximadamente de entre 750 y 1000 millones de dólares por año y los préstamos para abastecimiento de agua y drenaje de entre 3000 y 3500 millones.

Los efectos de las estrategias de autoconstrucción y de mejoramiento sobre las políticas nacionales de vivienda también fueron mínimos durante los años setenta, aunque existen pruebas de una mạyor influencia en los años recientes. Esto significa que el apoyo para proyectos de lotes y servicios y de mejoramiento se relaciona casi exclusivamente con el de los organismos internacionales. Un estudio de las políticas de vivienda tierra y asentamientos de 17 países en desarrollo, que adoptaron proyectos de lotes y servicios en los años setenta, revela que sólo Tanzania y Sudán los convirtieron en parte medular de sus políticas de vivienda urbana. ${ }^{11}$ Aunque algu nos países establecieron programas nacionales de lotes y servicios y mejoramiento de barriadas (esto es, Tailandia, Filipinas, Kenia y Brasil) su resultado, en general, fue reducido en relación con los crecientes déficits de vivienda.

El programa de Tailandia para 1979-1982, por ejemplo, tenía como objet ivo proporcionar únicamente 4000 pies de casa o lotes con servicios al año, mientras que, en Kenia, sólo $8 \%$ de las unidades de bajo costo incluidas en el Plan 1974-1978 fue terminado (a un costo cinco veces mayor que el previsto). ${ }^{12}$ En general, la mayoría de los gobiernos de los países en desa rrollo ha seguido dedicando la misma proporción de su PiB a la vivienda (entre 2 y $7 \%$ ) y las desigualdades en el gasto público para vivienda sólo se han reducido moderadamente desde el inicio de los setenta: "pocos paí-

${ }^{10}$ The Urban Edge, World Bank, a) julio de 1978; b) mayo de 1983; c) junio de 1979; d) no viembre de 1982; e) febrero de 1984; f) octubre de 1978.

${ }^{11}$ Hardoy y Satterthwaite (1981:254).

${ }^{12}$ Hardoy y Satterthwaite (1984:50). 
ses han logrado progresos significativos en el desarrollo de instituciones financieras que beneficien a un mayor número de pobres urbanos. Incluso en países como Túnez y Tailandia, que cuentan con instituciones financieras públicas y privadas bien establecidas, menos de $1 \%$ de la inversión institucional se destina al financiamiento de viviendas para grupos de bajos ingresos." ${ }^{13}$ En 1979, el Banco Nacional de la Vivienda (BNH), de Brasil, gastó aproximadamente $45 \%$ de sus inversiones en viviendas para grupos de medianos y altos ingresos. ${ }^{14}$ En El Salvador, el plan de desarrollo nacional 1979-1982 tenía como meta la producción de 50000 unidades casi exclusivamente para el $40 \%$ superior del espectro de distribución del ingreso, mientras que la producción de lotes y servicios se fijó en 17000 unidades, de las cuales fueron terminadas aproximadamente $10000 .^{15}$

Si se comparan esos niveles de gasto y sus resultados con las necesidades estimadas de viviendas y servicios, se obtiene una perspectiva incluso más grave. Así, se calcula que en el decenio 1972-1982 los proyectos de vivienda del Banco Mundial beneficiaron a 9 millones de personas. No obstante, la producción total de casas nuevas ( lotes y servicios y pies de casa) apenas alcanzó la cifra de 370000 unidades, que beneficiaron directamente a 2 millones de personas, mientras que 7 millones recibieron ayuda mediante proyectos de mejoramiento. ${ }^{16}$ Puesto que únicamente $60 \%$ de los beneficiarios pertenecían al grupo de menores recursos, tenemos que el número total de "pobres urbanos" que se beneficiaron durante ese periodo fue de sólo 5.4 millones.

¿Qué relación guardan esas cifras con las necesidades de vivienda? Desafortunadamente no existen datos disponibles sobre la demanda mundial de vivienda urbana en los años setenta; sin embargo, el Departamento de Asuntos Económicos y Sociales de las Naciones Unidas ${ }^{17}$ calcula que entre 1970 y 1980 se requerían 749 millones de unidades en las áreas urbanas y rurales para dar vivienda a la población de los países en desarrollo en la misma proporción de ocupación que en 1970 (aproximadamente una familia por morada).

Esto significa que habrían tenido que construirse 176 millones de unidades en los países en desarrollo durante ese decenio únicamente para hacer frente al aumento de población. Además, se estima que al menos $1 \%$ del

\footnotetext{
${ }^{13}$ Véase nota 10 (b).

14 Véase nota 10 (c).

${ }^{15}$ A. Harth Deneken, y M. Silva, "Mutual Aid and Progressive Development Housing: For What Purpose? Notes on the Salvadorean Experience", en P. M. Ward (ed.), Self-Help Housing: A Critique, Mansell, Londres, 1982, pp. 233-250.

${ }^{16}$ D. G. Williams, "International Agencies: The Role of the World Bank", en G. K. Payne (ed.), Low Income Housing in the Developing World..., op. cit., pp. 173-185.

${ }^{17}$ ONu, Global Review of Human Settlements, Centro para la Vivienda, la Construcción y la Planeación, Departamento de Asuntos Económicos y Sociales de la onu, Pergamon Press,
} Oxford, 1976. 
total de viviendas habría tenido que ser remplazado debido al envejecimiento, la renovación urbana, los terremotos, etc. Ello significa que habría sido necesario construir 67 millones de unidades, además de los 176 millones requeridos por motivos demográficos, por ende, construir un total de $243 \mathrm{mi}$ llones de unidades durante el decenio.

Podrían hacerse muchas objeciones a la manera como han sido calculadas estas cifras y al hecho de que incluyen el déficit de vivienda rural; sin embargo, incluso si redujésemos la cifra total en dos tercios para dejarla en 84 millones de unidades, y así dar cabida a las posibles críticas, sigue siendo evidente que el efecto positivo de los proyectos de lotes y servicios y de mejoramiento sobre los problemas de vivienda urbana en los países en desarrollo ha sido mínimo a pesar del optimismo de los defensores de la autoconstrucción. En 1985 se estimó que 100 millones de personas de los países en desarrollo no tenían vivienda de ningún tipo, y que aproximadamente 20 millones de niños y jóvenes vivían y dormían en las calles en A mérica Latina. ${ }^{18}$ Blitzer, Hardoy y Satterthwaite ${ }^{19}$ estiman que apenas $1 \%$ de la población urbana total de los países en desarrollo se ha beneficiado de los proyectos de vivienda, o de mejoramiento con financiamiento multilateral, durante los últimos veinte años.

Una comparación de las necesidades de vivienda para el decenio de 1970-1980 con los logros de los programas de lotes y servicios y de mejoramiento revela, también que más de $70 \%$ del déficit de viviendas estimado en los países en desarrollo fue producto de la necesidad de crear un "stock" de casas nuevas (en gran medida por motivos demográficos) y sólo $30 \%$ de la necesidad de remplazar la provisión de casas existentes. Sin embargo, prácticamente el total de los recursos desplegados ha sido destinado a mejo rar la provisión de viviendas existentes. Sólo una pequeñísima proporción de los recursos ha sido destinada al problema más importante de todos, que es la necesidad de crear una nueva provisión de viviendas. La razón de esto es evidente: el Banco Mundial estableció las condiciones de costo más alto y de capacidad de pago restringida para los proyectos de lotes y servicios virtualmente en todos los que se establecieron en los años setenta.

El verdadero meollo de la cuestión, como cada vez están más dispuesto s a admitirlo algunos funcionarios del Banco, lo constituyen los altos costos de los proyectos de lotes y servicios derivados de la necesidad de adquirir terrenos en el mercado, exponiendo así a los futuros residentes a todos los dramáticos efectos del proceso especulativo en el mercado urbano de bienes raíces.

También es cierto, que el volumen de fondos multilaterales disponibles para los programas de vivienda urbana se ve seriamente amenazado por

${ }^{18}$ UNCHs, Hábitat.

19 Véase la nota 2. 
las altas tasas de interés y la actitud de quienes se oponen a la ayuda en Estados Unidos y algunas partes de Europa. Los fondos totales puestos a la disposición de organismos como el Banco Mundial por los gobiernos miembros del mismo han sido reducidos y ello ha llevado ya a reducciones en los créditos "blandos" destinados a los países más pobres. Williams ${ }^{20}$ hace notar también que el Banco Mundial está poniendo un mayor énfasis en el desarrollo de la energía y la necesidad de proyectos de cofinanciamiento con el sector privado, y que la reciente incorporación de China reducirá aún más los fondos disponibles para proyectos urbanos destinados a los demás países miembros.

Tercera falacia: que los proyectos de autoconstrucción y de mejoramiento han beneficiado en gran medida a los grupos más pobres en las ciudades de los países en desarrollo

Aunque existen diferencias significativas entre los proyectos de lotes y servicios y los de mejoramiento, es a todas luces evidente que ambos han beneficiado-substancialmente a la clase urbana trabajadora, a los empleados gubernamentales y a los que trabajan en el "sector formal", antes bien que a los pobres urbanos. Más aún, parece ser que la participación de esos grupos ha ido en aumento a expensas de los "pobres urbanos" a partir de la terminación de los proyectos.

Desde principios de los años setenta, los principales organismos internacionales que hacen préstamos para vivienda urbana (incluyendo al Banco Mundial y a USAID) exigían una fuerte "orientación hacia los pobres" en los proyectos que tomaban en consideración para otorgar préstamos. En los proyectos debía identificarse una "población-objetivo", y la cantidad y calidad de los bienes y servicios entregados a través de los proyectos debían ajustarse estrechamente a las posibilidades de esa población-objetivo. En el caso de USAID, se definía como $50 \%$ inferior del espectro de distribución del ingreso, mientras que, en el del Banco Mundial, esa definición correspondía más comúnmente a $60 \%$ inferior.

Eso constituyó sin duda un progreso respecto a la situción previa, en la que la vivienda pública convencional no beneficiaba a los dos tercios inferiores del espectro de distribución del ingreso. ${ }^{21}$ No obstante, también es cierto que, debido a las enormes desigualdades en la distribución del ingreso de los países en desarrollo, la población-objetivo (el $60 \%$ inferior del es-

20 Vése la nota 16.

${ }^{21}$ D. Keare, y S. Parris, Evaluation of Shelter Programmes for the Urban Poor: Principal Findings, iBrD Staff Working Paper núm. 547, Banco Mundial, Washington, D. C., 1982. 
pectro) incluía seguramente una amplia gama de ocupaciones: desde los desempleados y los trabajadores del "sector informal", pertenecientes al estrato realmente inferior, hasta los artesanos, comerciantes en pequeño, los trabajadores urbanos y los empleados gubernamentales, pertenecientes al estrato superior. Y también es cierto que, dada la estructura del empleo urbano de la mayoría de los países en desarrollo y esas desigualdades en la distribución del ingreso, una proporción importante de la población cuenta sólo con un nivel mínimo de ingresos.

Los Informes de Evaluación de proyectos terminados del Banco Mundial, y algunos estudios realizados por observadores independientes indican que existe la tendencia a destinar los proyectos de lotes y servicios en particular (en los que el nuevo propietario tiene que comprar el lote) al nivel intermedio del espectro de distribución del ingreso. Y ése es precisamente el nivel ocupado por la clase trabajadora urbana, los empleados estatales y los estratos inferiores de la clase media. Amos, ${ }^{22}$ por ejemplo, hace notar que "ésa es una característica común a algunas juntas de desarrollo de vivienda en la India que no han logrado alcanzar las metas de lotes y servicios para los sectores económicamente más débiles de la comunidad y que, sin embargo, han logrado una alta producción para grupos más prósperos".

Los estudios sobre los proyectos de lotes y servicios en Lusaka indican altos niveles de empleo formal entre los participantes, lo cual es una importante razón de la baja incidencia de mano de obra en la autoconstrucción y del alto uso de mano de obra contratada para esos proyectos. ${ }^{23}$ Harth, Deneke y Silva ${ }^{24}$ señalan que en los proyectos financiados por el Banco Mundial para El Salvador "resultó virtualmente imposible beneficiar al 20\% más pobre de las familias urbanas [...] Los asentamientos resultaron más accesibles para las familias colocadas entre el tercero y el sexto niveles de ingresos".

Un estudio reciente de evaluación del Banco Mundial sobre cuatro proyectos de lotes y servicios en Senegal, Filipinas, El Salvador y Zambia indica que la siguiente fue una condición general de los proyectos: "La gran mayoría de los beneficiarios se encuentran en la mitad inferior de la distribu ción del ingreso, aunque algunos pueden tener ingresos tan altos como el $70^{\circ}$ percentil. La mayoría de los beneficiarios de los proyectos caen entre las categorías de los percentiles $20^{\circ}$ y $50^{\circ}$ de la distribución del ingreso, y no en los de más abajo." ${ }^{25}$ No obstante, el informe añade que "el beneficio de los más pobres de los grupos urbanos no ha sido un objetivo explíci-

${ }^{22}$ F. 1. C. Amos, "Political and Administrative Factors in Low Income Housing", en G. K. Payne, op. cit., pp. 161-171.

${ }^{23}$ C. M. Mbavu, "Learning from Lusaka", en E. Bruno, A. Korte y K. Mathey (eds.), Development of Urban Low Income Neighbourhoods in the Third World, Archimed Verlag, Darmstadt, 1984, pp. 287-304.

${ }^{24}$ Véase la nota 15

25 Véase la nota 21. 
to de los programas". ${ }^{26}$ Williams, funcionario del Banco Mundial, señala que los proyectos de lotes y servicios no son diseñados para atraer a los grupos de ingresos más altos, y que sus emplazamientos periféricos ofrecen pocas oportunidades de empleo a los que trabajan en el sector informal: "Por esta razón y debido a su gran tamaño, corren el riesgo de convertirse en 'ghettos' de obreros fabriles y de servidores públicos de bajo rango." 27

En el cuadro 2, que muestra la distribución del ingreso por familia en 14 proyectos de lotes y servicios en Asia, pueden encontrarse más pruebas de este fenómeno. Con tres excepciones (todas de la India) los datos muestran que sólo una minoría muy pequeña de las familias de esos proyectos tienen ingresos por abajo del nivel de subsistencia. Aunque aproximadamente entre 20 y $25 \%$ de las familias de esos proyectos se encuentra por lo general en esa situación, en algunos casos la proporción es muy baja -en el proyecto de reasentamiento de Simomulyo, en Surabaya, Indonesia, apenas $2.5 \%$ de todas las familias tenía ingresos por abajo del nivel de subsistencia. Con mucho, la mayor proporción de las familias de esos proyectos se encontraba en dos categorías: la correspondiente a aquellas familias con ingresos entre el nivel de subsistencia y dos veces ese nivel y la correspondiente a las familias con ingresos entre dos y cuatro veces el nivel de subsistencia. Sólo en cuatro de los 14 proyectos (tres en la India y uno en Indonesia) había menos de $50 \%$ de las familias con ese nivel de ingresos, y en seis proyectos más de tres cuartas partes de las familias se encontraban a ese nivel de ingresos.

Por supuesto, es precisamente a ese nivel donde se encuentra la mayoría de la clase urbana trabajadora y la de los que trabajan en el sector "formal" capitalista. Cabe observar también, conforme se indica en el cuadro 2, que en algunos casos (los proyectos de Calcuta y Yakarta) se encuentra presente también una proporción importante de familias con ingresos mayores a cuatro veces el ingreso de subsistencia, lo que indica la presencia de grupos de ingresos medios superiores en algunos de esos proyectos.

El mismo estudio ${ }^{28}$ indica que esa tendencia a que los proyectos de lotes y servicios sean destinados a grupos de medianos ingresos es una característica general de los proyectos desarrollados por la Autoridad Nacional de la Vivienda (ANV) (National Housing Authority: NHA), de Tailandia. Aunque la población-objetivo fue definida como aquellas familias con ingresos inferiores a 3500 bahts $^{29}$ al mes "es probable que muchas más familias del nivel de 4000 bahts solicitarán lotes ANV que las familias con in-

26 Idem.

27 Véase la nota 16.

${ }^{28}$ P. J. Swan, A. Wegelin y K. Panchee, The Management of Sites and Services Housing Schemes: The Asian Experience, John Wiley and Sons, Chichester, 1983.

${ }^{29}$ Unidad monetaria de Tailandia. (N. del T.) 


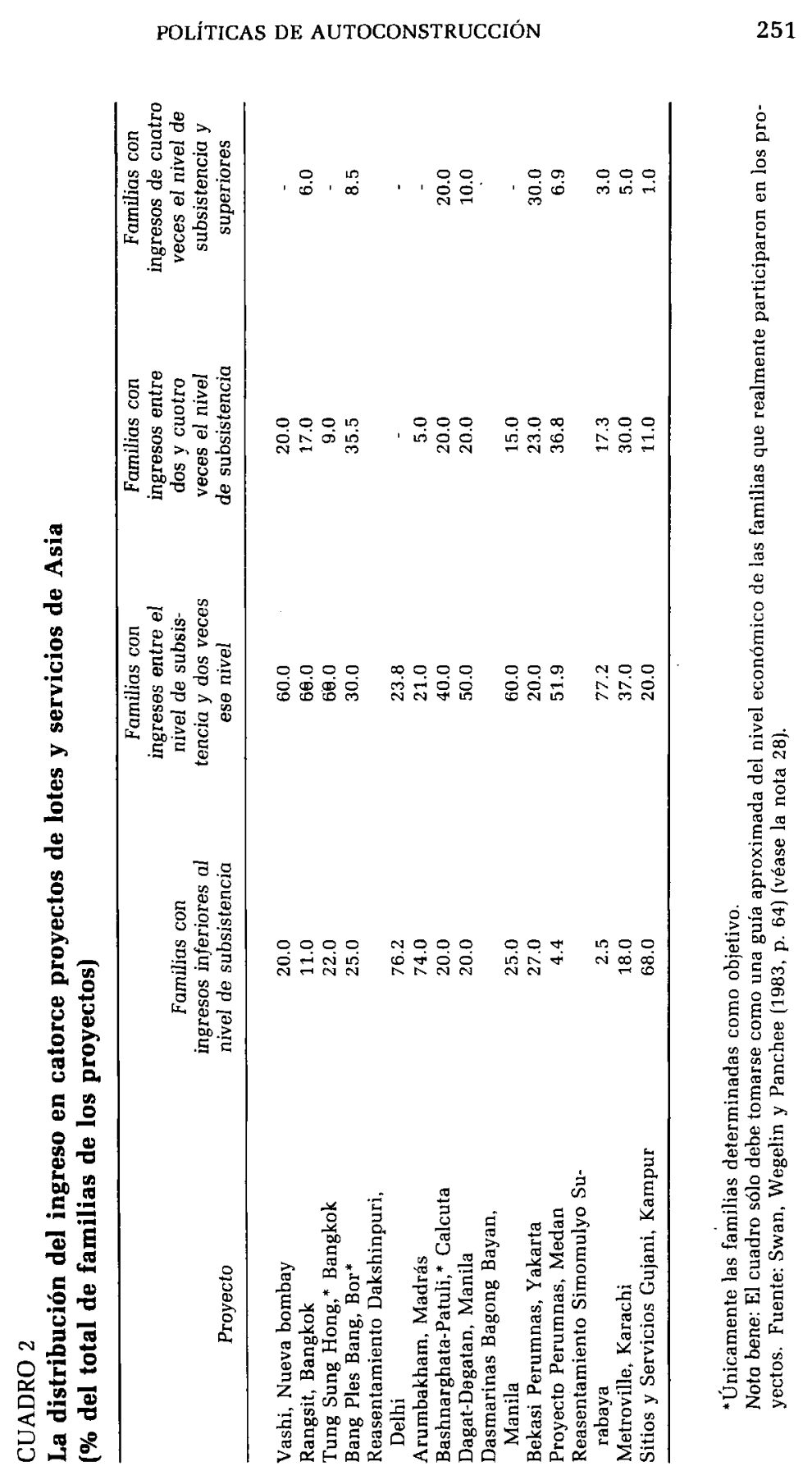


gresos de alrededor de 2500 bahts". En el proyecto de lotes y servicios Tung Song, en Bangkok, $84 \%$ de los solicitantes tenía ingresos de alrededor de 3000 bahts al mes y $48 \%$ contaba con ingresos de alrededor de 4000 bahts al mes. ${ }^{30}$

No es sorprendente el que los proyectos de mejoramiento de tugurios y asentamientos irregulares hayan sido más eficaces que los de lotes y servicios para beneficiar a las familias de ingresos muy bajos. La índole multiclasista de esos asentamientos ha sido establecida desde hace mucho tiempo en la literatura, por lo que no es sorprendente tampoco el que las mejoras introducidas en la forma de proyectos de mejoramiento hayan beneficiado a una gama más amplia de grupos de ingresos que las introducidas en los proyectos de lotes y servicios. Williams ${ }^{31}$ afirma que esos proyectos de mejoramiento han beneficiado incluso al grupo de ingresos correspondientes a la percentila ${ }^{32} 10^{\text {a }}$ (lo cual, debe admitirse, excluye todavía a una proporción importante de la población urbana en algunas ciudades).

No obstante, dada la importancia de esos asentamientos como centros de residencia para la clase trabajadora urbana, es evidente que deben beneficiarse muy considerablemente de ese tipo de proyecto. Schlyter, ${ }^{33}$ por ejemplo, estima que a mediados de los años setenta tres cuartas partes de los miembros de la fuerza de trabajo residentes en los asentamientos mejorados de George, en Lusaka, tenían empleo en el "sector formal".

Con todo, no se sabe si para los proyectos de mejoramiento ha habido una selección preferencial de asentamientos con una alta proporción de trabajadores del sector capitalista organizado - motivada quizá por su mayor influencia política- por lo que sin duda es necesario investigar más a fondo esta cuestión. No obstante, el no poder identificar un mecanismo discriminatorio consciente no descalifica necesariamente el argumento, porque en los criterios de capacidad de pago y recuperación de costos incluidos en el proceso de planeación de los proyectos está implícita una tendencia a destinar esos proyectos a aquellos que trabajan en el sector formal organizado, y los organismos internacionales insisten en esos criterios.

Además, las mismas exigencias de capacidad de pago y recuperación de costos han conducido de manera creciente a una situación en la que las autoridades nacionales de la vivienda y sus patrocinadores internacionales se han visto obligados a aumentar la proporción de lotes con mayores servicios (a menudo con pies de casa) destinados a grupos de medianos ingresos en proyectos específicos de lotes y servicios. Así, a partir de los años

30 Véase la nota 28.

31 Véase la nota 16

${ }^{32}$ Número correspondiente a una de 100 divisiones iguales del campo de valores de una estadística en una muestra dada, y que caracteriza un valor contenido de la estadística que no es sobrepasado por un porcentaje indicado de todos los valores de la muestra. (N. del T.)

${ }^{33}$ A. Schlyter, Upgrading Reconsidered-The George Studies in Retrospect, The National Swedish Institute for Building Research, Lund, Sweden, 1984. 
ochenta se hizo práctica común en los proyectos de ese tipo patrocinados por el Banco Mundial, el ofrecer una amplia gama de tamaños de lotes y redes de servicios a precios diferentes.

El proyecto Arumbakham de Madrás, por ejemplo, ofrecía cinco diferentes tamaños de lotes, que iban de 40 a $223 \mathrm{~m}^{2}$, con diversos grados de desarrollo de los servicios, lo que proporcionaba un total de seis opciones. ${ }^{34}$ En proyectos de Tailandia, Filipinas, Indonesia, México y varios países africanos se ha introducido una gama similar de opciones de terrenos. Aunque se ha justificado esta política en función de la necesidad de impedir los asentamientos de una sola clase y de preservar los efectos benéficos que los ingresos de las clases medias ejercen sobre las estrategias de supervivencia de los grupos de bajos ingresos, su propósito real ha sido el generar subsidios que pasen de un proyecto a otro (subsidios cruzados) en vista de la escalada de los costos de la tierra y los materiales de construcción y de las crecientes tasas de interés.

Enfrentadas a la alternativa de reducir los servicios y las superficies de los lotes para los grupos de bajos ingresos a niveles intolerables, las autoridades se han visto cada vez más forzadas a dirigir una proporción creciente de los recursos de los proyectos a aquellos que trabajan en el sector capitalista "formal" para no desviarse demasiado de la meta global de recuperación total de los costos. A falta de medidas eficaces para hacer frente a esa escalada de los costos, y dado el creciente problema de vivienda de la clase media, existen serias razones para creer que las medidas adoptadas respecto a los subsidios cruzados constituyen el punto de partida para dejar de beneficiar a los más pobres, y que una proporción creciente de los recursos de esos proyectos será destinada a los grupos relativamente más ricos.

La cuestión sobre qué clases sociales han sido beneficiadas mediante los proyectos de lotes y servicios y de mejoramiento también debe ser relacionada con el fenómeno de la "usurpación de la clase media", del cual existen numerosos informes, que ha sido provocado por los aumentos de los precios de la tierra y de los alquileres en esos asentamientos y por la severidad de la escasez de vivienda para los grupos de clase media.

Quizá uno de los más graves errores de los defensores de la autoconstrucc ión, en la interpretación que hacen de la estructura residencial urbana, es la idea de que la movilidad residencial se detuvo una vez que el inquilino del interior de la ciudad llegó al asentamiento irregular de la periferia. ${ }^{35}$

Durante los años setenta se acumuló una cantidad cada vez mayor de

34 Véase la nota 28.

35 R. Burgess, “Ideology and Urban Residential Theory in Latin America”, en D. T. Herbert, y R. J. Johnston (eds.), Geography and the Urban Environment: Progress in Research and Applications, John Wiley and Sons, Chichester, vol. 4, 1981, pp. 57-114. 
pruebas que muestran, por el contrario, los altos niveles de rotación residencial en los asentamientos irregulares. ${ }^{36,}{ }^{37,}{ }^{38,}$, 39 La legalización de la propiedad (posesión-tenencia), los crecientes costos de la tierra y la incapacidad para soportar la carga de los impuestos sobre la tierra y la propiedad han provocado una emigración de los habitantes más pobres de los asentamientos irregulares, así como una inmigración de residentes más acomodados ansiosos de comprar la tierra recién regularizada. A menudo se da el caso de que los ocupantes irregulares originales retornen al sistema urbano a través de nuevas ocupaciones irregulares, y también se tienen amplios informes sobre la existencia de ocupantes irregulares "profesionales".

La importancia de este fenómeno para la nueva estrategia de los proyectos de lotes y servicios y de mejoramiento también fue señalada, a mediados de los años setenta, a un nivel teórico:

Si el estado legaliza post facto una invasión otorgando la posesión (como en los proyectos de mejoramiento) no hará sino facilitar el establecimiento de relaciones de conveniencia en asentamientos donde hasta entonces no existían o donde su desarrollo era mínimo (precisamente porque eran ilegales). Si legaliza tales asentamientos antes de la posesión (como en los proyectos de lotes y servicios) no hará sino permitir la valoración de mercado de los terrenos, los cuales, de otra manera, habrían sido invadidos o comprados ilegalmente (a precios más bajos). En ambos casos, la intervención del estado (...) ha facilitado el establecimiento de la relación de conveniencia en los asentamientos. ${ }^{40}$

Ahora empiezan a comprenderse cada vez más las perturbadoras consecuencias de la integración de los grupos de bajos ingresos al mercado de la tierra mediante proyectos de lotes y servicios y de mejoramiento, al igual que los efectos sobre la composición de clases de esos asentamientos.

La experiencia obtenida de muchos proyectos de mejoramiento es que las mejoras mismas introducidas en áreas pobres e irregulares han tenido un marcado efecto sobre el valor de la tierra, los precios de las casas y los alquileres. Baross, ${ }^{41}$ por ejemplo, informa que, en Yakarta, los valores de la propiedad en aldeas mejoradas aumentaron en promedio $1771 \mathrm{rupias} / \mathrm{m}^{2}$ más que en las aldeas no mejoradas y que, en Surabaya, la diferencia fue

${ }^{36}$ P. M. Ward (ed.), Self-Help Housing: A Critique, Mansell, Londres, 1982.

${ }^{37}$ S. E. Eckstein, The Poverty of Revolution: The State and the Urban Poor in Mexico, Princeton University Press, Princeton, 1977.

${ }^{38}$ W. A. Cornelius, Politics and the Migrant Poor in Mexico City, Stanford University Press, Stanford, 1975.

${ }^{30}$ P. Connolly, “Uncontrolled Settlements and Self-Build: What Kind of Solution?", en Ward, P. M., op. cit.

${ }^{40} \mathrm{R}$. Burgess (1979).

${ }^{41}$ P. Baross, "Kampong Improvement or Kampong Development-Appraisal of the Low Income Settlement Policy in Indonesia", en F. Bruno, A. Korte y K. Mathey (eds.) Development of Low Income Settlement Upgrading Policy in Indonesia, Archimed Verlag, Darmstadt, 1984. 
aún mayor, esto es, 2175 rupias $/ \mathrm{m}^{2}$. Además, el alquiler promedio en las aldeas de Surabaya aumentó en 50 y $100 \%$ a partir de que se hicieron las mejoras, y los alquileres aumentaron hasta en $250 \%$ en lugares donde se habían construido caminos para vehículos. En Calcuta, "los alquileres en los tugurios mejorados han aumentado significativamente en comparación con los aumentos en los asentamientos no mejorados". ${ }^{42}$

Paralelamente a los proyectos de lotes y servicios se han desarrollado mercados de tierras y viviendas muy activos y, en realidad, en "muchos países africanos, del medio oriente y del pacífico [esos proyectos] han provocado el establecimiento de mercados muy activos con consecuencias potencialmente desastrosas en el largo plazo". ${ }^{43}$ Además, también se han observado aumentos significativos en el peso de los cargos por servicios públicos y los gravámenes sobre la propiedad tanto en el caso de los proyectos de lotes y servicios como en los de mejoramiento.

Skinner ${ }^{44}$ señala que el repago de 14 millones de dólares por mes exigido por electrificación doméstica y alumbrado público en el "pueblo joven" Villa El Salvador, en Lima, representaba aproximadamente $30 \%$ de los ingresos mensuales promedio. A finales de los años setenta se estimó que los gravámenes sobre la propiedad en los proyectos de mejoramiento de aldeas en Yakarta aumentaban a una tasa de $15 \%$ anual. ${ }^{45}$ Junto con la escasez de viviendas para los grupos de ingresos medios-bajos y medios, esos fenómenos han tenido un efecto significativo en la composición de clases del asentamiento, forzando a los grupos de ingresos más bajos ya sea a abandonar el asentamiento, ya a apiñarse en él cada vez más adaptándose a los alquileres.

Cohen, ${ }^{46}$ por ejemplo, observa que "en algunos programas de mejoramiento los aumentos en los cargos mensuales dieron por resultado aumentos en los alquileres, lo cual, a su vez, provocó presiones sobre los pobres, que tuvieron que abandonar el vecindario (como en África) o hacinarse en el mismo cuarto (como en Indonesia)". En el proyecto de lotes y servicios de Vashi, en las afueras de Bombay, se seleccionó originalmente una población-objetivo en la que $60 \%$ de los beneficiarios provenía de los "sector es económicamente débiles" o de los "grupos de ingresos bajos" de los tugurios y asentamientos irregulares de la ciudad.

Después de haber sido puesto en práctica, se observó que "aproximada mente $75 \%$ de las casas del proyecto habían sido revendidas". ${ }^{47}$ Los auto-

42 Véase la nota 10 (d).

${ }^{43}$ Véase la nota 3.

${ }^{44}$ R. Skinner, "Self-Help, Community Organization and Politics: Villa El Salvador, Lima", en P. M. Ward, op. cit.

46 The Wall Street Journal, noviembre de 1977.

46 Véase la nota 8.

${ }^{47}$ Véase la nota 28. 
res mencionan las dificultades de los repagos mensuales, los elevados cargos por servicios públicos y los gravámenes sobre la propiedad como las razones principales de ese proceso de "mercantilización". También se ha consignado ampliamente el crecimiento del alquiler y el subalquiler de propiedades en los proyectos en América Latina, África y Asia. En el Informe de Evaluación de Lusaka ${ }^{48}$ del Banço Mundial, se hace notar que "existen motivos para temer que los sectores más pobres serán explotados, ya sea por tener que pagar un alquiler excesivo, ya por tener que excluirlos del proyecto". A finales de los años setenta, se informó que en el proyecto del Valle Mathare de Nairobi los que no pertenecían a la "clase media" estaban preparados para ofrecer alquileres de cinco a diez veces más altos que la tasa corriente, mientras que también existen informes en el sentido de que en el proyecto Dandora se dio el caso de ventas secretas de lotes a especuladores, quienes estaban construyendo casas de huéspedes para alquiler en esos lotes. ${ }^{49}$

Las restricciones iniciales impuestas por las autoridades nacionales y sus patrocinadores internacionales a la venta y alquiler de propiedades han sido reemplazadas ahora por una actitud más relajada respecto a la asignación, y la práctica de prestar para facilitar el subarrendamiento se ha transformado en un cuerdo y cada vez más común plan de acción de la política predominante.

Cualesquiera que sean las demás cuestiones que intervienen, los efectos sobre la composición de clases de los asentamientos son significativos:

...esa incorporación en los mercados de tierras y bienes inmuebles de una ciudad beneficia en particular a los grupos de ingresos superiores y medios y a algunos miembros del nivel superior de los grupos de bajos ingresos, pero aísla aún más a los grupos más pobres de las oportunidades de vivienda y empleo adecuados. $^{50}$

Un tercer efecto sobre la composición de clases de los proyectos de lotes y servicios ha sido su emplazamiento. En un esfuerzo por reducir los costos de la tierra, los proyectos han sido ubicados a menudo en la periferia urbana, a distancias considerables de las principales fuentes de empleo informal. En la India y en el este de Asia, ello ha provocado que los pobladores irregulares excluidos de los proyectos de mejoramiento de las zonas centrales de las ciudades hayan sido ubicados a una distancia de entre 20 y $30 \mathrm{~km}$ del centro, lo cual, a su vez, impone un excesivo costo de transporte sobre los presupuestos familiares (en Manila representó hasta $30 \%$ del

${ }^{48}$ Banco Mundial, Evaluation of Sites and Services Projects: The Experience from Lusaka, Zambia, World Bank Staff Working Paper núm. 548, Washington, D. C., 1982.

49 The Guardian, 14 de diciembre de 1977.

${ }^{50}$ Véase la nota 3 . 
salario mínimo). Así, muchas familias de ingresos inferiores se han visto obligadas a vender a los que cuentan con un empleo más regular y a seguir ocupando asentamientos irregulares. La creciente téndencia a emplazar la industria en la periferia urbana también ha reforzado el atractivo de los proyectos para la clase trabajadora urbana y para aquellos que trabajan en el sector capitalista "formal".

Cuarta falacia: que los proyectos de lotes y servicios y de mejoramiento se caracterizan principalmente por la utilización de mano de obra familiar no remunerada (esto es, mano de obra para la autoconstrucción)

Según Portes y Walton, ${ }^{51}$ "el sector formal saca provecho de vastas cantidades de mano de obra no remunerada" derivada de las actividades de autoconstrucción de sus trabajadores que viven en asentamientos irregulares, en fraccionamientos ilegales y en los proyectos de lotes y servicios y de mejoramiento; esto provoca que no exista relación alguna entre niveles de salarios y alquileres en el mercado de viviendas regulado.

Se puede estar de acuerdo, por una parte, en el hecho de que toda mano de obra utilizada en la autoconstrucción es una mano de obra no remunerada que representa una extensión de la jornada laborable y, por la otra, en que todos los programas para solucionar el problema de la vivienda antes mencionados implican la utilización de cantidades más o menos grandes de mano de obra en la autoconstrucción. Sin embargo, la idea de que esas estrategias de vivienda incluyen "vastas cantidades" de mano de obra familiar no remunerada debe ser puesta en tela de juicio en todos los casos y, en particular, en el dé los proyectos estatales de autoconstrucción y mejoramiento de viviendas.

Un exameń cuidadoso de la naturaleza del proceso de la mano de obra en todas esas actividades relacionadas con la vivienda revela que la reducción én los costos de las viviendas se logra, no tanto a través del empleo de la mano de obra familiar no remunerada, sino más bien de la utilización de mano de obra asalariada, no sindicalizada, especializada y no especializada, contratada en el sector "informal" de la construcción.

A mediados de los años setenta, un buen número de observadores, ${ }^{52,} 53,54$ hizo notar por primera vez a un nivel teórico la gran discre-

${ }^{51}$ A. Portes, y J. Walton, Labor, Class and the International System, Nueva York, Academic Press, 1981.

52 Pradilla (1974).

${ }^{53} \mathrm{H}$. Molina, La vivienda y el problema de la vivienda en Colombia, SIAP-CIID-CPU, Bogotá, 1976

${ }^{54} \mathrm{R}$. Burgess, "Petty Commodity Housing or Dweller Control? A Critique of John Turner's Views on Housing Policy", en World Development, vol. 6, núm. 9/10, 1978, pp. 1105-1133. 
pancia existente entre las descripciones que hacen los defensores de la autoconstrucción del proceso de trabajo, presente en los asentamientos urbanos de grupos de menores ingresos, y la verdadera naturaleza de ese proceso de trabajo. Recientemente, la atención se ha enfocado a las diferencias entre el proceso de trabajo presente en los asentamientos irregulares y el que se da en los proyectos estatales de autoconstrucción de viviendas, así como en la importancia de esas diferencias para el costo de las casas producidas. ${ }^{55}$ Además, se cuenta ya con una gran cantidad de datos empíricos, particularmente sobre América Latina y el este de Asia, que revelan la limitada importancia que tiene la autoconstrucción en los asentamientos irregulares y fraccionamientos ilegales.

En general, esos datos muestran que la autoconstrucción se limita al desempeño de un reducido número de tareas no especializadas, destinadas en parte a la estructura básica y en parte a los acabados. La mayoría de la mano de obra especializada que implica la colocación de cimientos, construcción de muros y montaje de instalaciones se adquiere mediante la contratación de obreros especializados y semiespecializados que, en ocasiones, operan en grupos en los mercados informales de mano de obra que existen en el sector de la construcción. Sin embargo, una diferencia importante que todavía subsiste entre los procesos de construcción que se llevan a cabo en asentamientos irregulares y los que se presentan en los proyectos estatales de autoconstrucción de viviendas es el hecho de que el propietario sigue actuando como su propio diseñador, arquitecto, contratista y supervisor en todas las etapas del proceso de construcción.

En un estudio reciente sobre la ciudad de México, Ward ${ }^{56}$ descubrió que 31,61 y $73 \%$ de los propietarios interrogados en tres asentamientos irregulares habian utilizado alguna forma de mano de obra remunerada en la construcción de sus hogares: en ocasiones se aplicó el sistema de producción por encargo, utilizando contratistas, en otras se utilizó parcialmente la mano de obra remunerada -en los casos en que una parte de la vivienda fue autoconstruida- y, en otras más, sólo el trabajo remunerado. El estudio de Moser sobre el asentamiento irregular Indio Guayas, en Guayaquil, ${ }^{57}$ reveló el uso muy extendido de mano de obra remunerada en la construcción de viviendas, de las que el $25 \%$ fue producido por encargo por grupos de trabajadores que construían bajo contrato para el propietario.

La naturaleza del proceso de trabajo en los proyectos de lotes y servicios y de mejoramiento ha sido menos estudiada, y sigue constituyendo una

${ }^{55}$ R. Burgess, "The Limits of State Self-Help Housing Programmes", in Development and Change, vol. 16, núm. 2, abril de 1985, pp. 271-312.

${ }^{56}$ Véase la nota 36.

${ }^{57}$ C. Moser, "A Home of One's Own: Squatter Housing Strategies in Guayaquil, Ecuador", en A. G. Gilbert, y otros (eds.), Urbanization in Contemporary Latin America, John Wiley and Sons, Chichester, 1982, pp. 159-190. 
prioridad de la investigación, dada su importancia para un gran número de debates teóricos significativos respecto a la función del estado y a la organización de los modos de producción. No obstante, a partir de los datos empíricos que han surgido de esos proyectos desarrollados en los años setenta, es evidente no sólo que la autoconstrucción es mínima, sino también que es mucho menor que la que se da en los procesos de construcción presentes en los asentamientos irregulares.

Primero, resulta que, casi sin excepción, los proyectos han consistido en que el organismo que los pone en práctica contrata pequeñas compañías constructoras que hacen la entrega inicial de lotes y servicios, con o sin núcleos sanitarios, y/o pies de casa. En un reducido número de casos, aparentemente en disminución, la comunidad ha participado de alguna manera en la autoconstrucción colectiva para la instalación de servicios básicos (por ejemplo, cavado de cepas y tendido de tuberías) pero ello ha sido excepcional.

En años recientes, el Banco Mundial ha buscado incrementar la participación del sector privado a través de sus políticas de préstamos, en lo que se ha visto influido, sin duda, por las actitudes del actual gobierno estadunidense: "El Banco se está desplazando del anterior concepto de asistencia técnica, según el cual a la gente se le enseñaba realmente cómo colocar un ladrillo sobre otro, hacia el estímulo a las soluciones de libre mercado." 58 En algunos casos (Marruecos y Filipinas, por ejemplo) ello ha llevado al banco a poner algunos préstamos directamente a la disposición de compañías constructoras privadas para la construcción de proyectos privados de lotes y servicios sobre la base de la obtención de utilidades.

Keare y Parris $^{59}$ han identificado cuatro tipos de producción utilizados en los proyectos financiados por el Banco Mundial, a los que puede añadirse uno más. En todos los casos, la construcción sigue a la entrega de lotes y servicios que hacen los contratistas, aunque en unos cuantos de los proyectos se ha aplicado el sistema de autoconstrucción colectiva de los beneficarios para la instalación de servicios. Esos tipos son:

1. Autoconstrucción, en la que la familia utiliza su propia mano de obra para construir la casa.

2. Construcción de ayuda mutua, en la que las familias trabajan juntas en grupos para construir la casa de cada quien con la supervisión de los ad ministradores del proyecto. Este tipo de construcción puede extenderse al aprovisionamiento de instalaciones comunitarias.

3. Producción, en la que la familia requiere a un contratista para construir la vivienda (producción por encargo).

58 Véase la nota 10 (e).

59 Véase la nota 21. 
4. Producción, en la que la familia contrata su propia mano de obra para construir la casa, y supervisa a cada uno de los trabajadores.

5. Autoconstrucción colectiva, en la que se organizan grupos de trabajo entre la comunidad para construir escuelas, puestos médicos, salas de reuniones, instalaciones recreativas, etcétera.

La construcción a través de la ayuda mutua ha sido poco utilizada en los proyectos de lotes y servicios (con excepción de los proyectos FUNDASAL en El Salvador) y el Banco Mundial la desalienta activamente por considerarla ineficaz y difícil de organizar. El uso de la autoconstrucción colectiva ha variado de proyecto a proyecto, y el Banco Mundial la desalienta en algunos casos (Lusaka, por ejemplo); en otros, se vale de las estructuras de desarrollo comunitario institucionalizadas para alentarla (Colombia y Bolivia). Pero todos los informes de evaluación de los proyectos de lotes y servicios existentes, al igual que los de observadores independientes, coinciden en el hecho de que muy pocos de los beneficiarios de los proyectos han construido completamente sus casas utilizando su propia mano de obra.

La forma más común ha consistido en que los residentes contraten trabajadores especializados o semiespecializados (albañiles para los cimientos y los muros, carpinteros para los techos y las puertas, etc.), ya sea con o sin contribución de autoconstrucción para las tareas no especializadas. También se consigna ampliamente la construcción de viviendas por encargo, mediante contrato, aunque es menos común.

Así, el Informe de Evaluación de Lusaka ${ }^{60}$ del Banco Mundial afirma que "en una proporción más alta que lo esperado, las familias decidieron contratar mano de obra, antes bien que construir por sí mismas". El informe recomienda también que, en los programas futuros, la ayuda mutua no debería ser una condición requerida para el proceso de construcción.

Esto parece ser una manifiesta subestimación de la escala del fenómeno, ya que, respecto a los mismos proyectos, $\mathrm{Mbavu}^{61}$ hace notar que "en realidad, muy pocos de los participantes en los programas de zonas sobrepobladas y de lotes y servicios construyeron sus casas utilizando únicamente su propia mano de obra. Un $80 \%$ contó con mano de obra contratada para los trabajos especializados". Indica que, en el proyecto Chawama, $80 \%$ de las viviendas fueron construidas con mano de obra contratada, $12 \%$ por los propietarios y $8 \%$ por parientes y ninguna casa fue construida por grupos de familias.

En los proyectos de El Salvador, "muchas familias de ingresos superiores o más especializadas prefieren diseñar y construir por sí mismas, uti-

60 Véase la nota 48.
61 Véase la nota 23. 
lizando a menudo contratistas menores o mano de obra no especializada remunerada". ${ }^{62}$ En un proyecto, $40 \%$ de las familias emplearon contratistas o mano de obra remunerada. En el Programa de Vivienda Madrás 19781984, cuyo objetivo consistía en proporcionar lotes y servicios, pies de casa y servicios municipales para 30000 familias, se descubrió que "la mayoría de la gente construyó sus casas en menos de un año, a menudo utilizando contratistas para hacer el trabajo". ${ }^{63}$ También se cuenta con informes similares sobre proyectos en Mali, Costa de Marfil, Kenia, Filipinas y México. El cuadro que surge de los proyectos de mejoramiento es parecido, ya que los préstamos destinados a ese fin se utilizan para contratar mano de obra remunerada; además se experimentan grandes dificultades en la organización de la autoconstrucción colectiva para el mantenimiento y mejoramiento de las instalaciones existentes (Yakarta y Calcuta, por ejemplo).

Tal ha sido la escala de este fenómeno, que el Banco Mundial ya introdujo una política, conforme a la cual, los préstamos que se hicieron previamente a los residentes de los proyectos para la compra de materiales de construcción pueden ser utilizados, de manera oficial, para contratar mano de obra.

Se puede concluir, entonces, que se ha exagerado mucho la importancia de la autoconstrucción no remunerada en los proyectos de sitios y servicios y de mejoramiento. Las pruebas empíricas revelan que los tan alabados proyectos de autoconstrucción de viviendas patrocinados por el e stado y los organismos internacionales se ubican, en efecto, tanto en el sector capitalista "formal" de la construcción (a través de la entrega de lotes con servicios por parte de contratistas) como en los mercados de mano de obra del sector "informal" de la construcción.

Si se logra su abaratamiento es, no tanto por el uso de mano de obra familiar no remunerada, sino por la amplia explotación de una mano de obra asalariada no sindicalizada a la que se le pagan tasas muy por abajo del salario mínimo legal. La extensión de esa explotación se expresa en la reducida diferencia de costos que existe entre la vivienda construida enteramente mediante la autoconstrucción y la construida por contratistas. El Banco Mundial, por ejemplo, estima que, en El Salvador, la ayuda mutua pudo reducir los costos de las viviendas en sólo un $10 \%$ en relación con las viviendas construidas por contratistas. ${ }^{64}$ Consecuentemente, vale la pena reflexionar sobre cómo pudo ocurrir que la "libertad para construir" haya llegado a significar "libertad para contratar", y cómo puede justificarse el bienestar social de los pobres en función de la explotación de los más pobres.

\footnotetext{
62 Véase la nota 10 (f).

63 Véase la nota 10 (d).

64 Véase la nota 10 (f).
} 


\section{Bibliografía}

Amos, F. J. C., 1984. "Political and Administrative Factors in Low Income Housing”, en Payne, G. K., (ed.), Low Income Housing in the Developing World - The Role of Sites and Services and Settlement Upgrading, John Wiley and Sons, Chichester, pp. 161-171.

Banco Mundial, 1973-1984. Annual Reports, Washington, D. C. The Urban Edge, julio de 1978 (a); octubre de 1978 ib); junio de 1979; noviembre de 1982; mayo de 1983 y febrero de 1984.

1982. Evaluation of Sites and Services Projects: The Experience from Lusaka, Zambia, World Bank Staff Working Paper núm. 548.

Baross, P. 1984. "Kampong Improvement or Kampong Development - Appraisal of the Low Income Settlement Policy in Indonesia", en Bruno, F., A. Korte y K. Mathey (eds.), Development of Low Income Settlement Upgrading Policy in Indonesia, Archimed Verlag, Darmstadt, 1984.

Blitzer, S., J. E. Hardoy y D. Satterthwaite, 1983. "The Sectorial and Spatial Distribution of Multilateral Aid for Human Settlements", en Habitat International, vol. 7 , núm. 1/2, 1983, pp. 103-127.

Burgess, R. 1978. "Petty Commodity Housing or Dweller Control? A Critique of John Turner's Views on Housing Policy", en World Development, vol. 6, núm. 9/10, pp. 1105-1133.

, 1981. "Ideology and Urban Residential Theory in Latin America", en Herbert, D. T. y R. J. Johnston (eds.), Geography and the Urban Environment: Progress in Research and Applications, John Wiley and Sons, Chichester, vol. 4, pp. 57-114.

1985. "The Limits of State Self-Help Housing Programmes", en Development and Change, vol. 16, núm. 2, pp. 271-312.

1987. Labour, Shelter and Global Capitalism, Metheum, Londres.

Cohen, M. A., 1983. The Challenge of Replicability: Towards a New Paradigm for Urban Shelter in Developing Countries, World Bank Reprint Series núm. 287, World Bank, Washington, D. C.

Connolly, P. 1982. "Uncontrolled Settlements and Self-Build: What Kind of Solution?", en Ward, P. M., (ed.), en Self-Help Housing: A Critique, Mansell, Londres.

Cornelius, W. A., 1975. Politics and the Migrant Poor in Mexico City, Stanford University Press, Stanford.

Eckstein, S. E., 1977. The Poverty of Revolution: The State and the Urban Poor in Mexico, Princenton University Press, Princeton.

Harth Deneke, A. y M. Silva, 1982. "Mutual Aid and Progressive Development Housing: For What Purpose? Notes on the Salvadorean Experience", en Ward, P. M., (ed.), Self-Help Housing: A Critique, Mansell, Londres, pp. 233-250.

Keare, D. y S. Parris, 1982. Evaluation of Shelter Programmes for de Urban Poor: Principal Findings, IBRD Staff Working Paper núm. 547, Banco Mundial, Washington D. C.

Mbavu, C., 1984. "Learning from Lusaka", en Bruno, E., A. Korte y K. Mathey (eds.), Development of Urban Low Income Neighbourhoods in the Third World, Archimed Verlag, Darmstad, pp. 287-304

Molina, H., 1976. La vivienda y el problema de la vivienda en Colombia, SIAP-CIIDCPU, Bogotá.

Moser C., 1982. "A Home of One's Own: Squatter Housing Strategies in Guayaquil, Ecuador", en Gilbert, A. G., et al. (eds.), Urbanization in Contemporary Latin America, Chichester, John Wiley and Sons, pp. 159-190 
ONU, 1976. Global Review of Human Settlements, Centro para la Vivienda, la Construcción y la Planeación, Departamento de Asuntos Económicos y Sociales de la ONU, Pergamon Press, Oxford.

Payne, G. K., (ed.), 1984. Low Income Housing in the Developing World - The Role of Sites and Services and Settlement Upgrading, John Wiley and Sons, Chichester, 1984.

Portes, A. y J. Walton, 1981. Labour, Class and the International System, Nueva York, Academic Press.

Schlyter, A., 1984. Upgrading Reconsidered - The George Studies in Retrospect, The National Swedish Institute for Building Research, Lund, Suecia.

Skinner, R., 1982. "Self-Help, Community Organization and Politics: Villa El Salvador, Lima", en Ward, P. M., (ed.), Self-Help Housing: A Critique, Mansell, Londres.

Swan, P. J., A. Wegelin y K. Panchee, 1983. The Management of Sites and Services Housing Schemes: The Asian Experience, John Wiley and Sons, Chichester.

Ward, P. M., (ed.), 1982. Self-Help Housing: A Critique, Mansell, Londres.

Williams, D. G., 1984. "International Agencies: The Role of the World Bank", en Payne, G. K., (ed.), Low Income Housing in the Developing World - The Role of Sites and Services and Settlement Upgrading, John Wiley and Sons, Chichester, pp. 173-185. 
